\title{
INVERSION FOR THE INPUT HISTORY OF A DYE TRACING EXPERIMENT
}

\author{
Malcolm S. Field ${ }^{1}$ And Guangquan Li²*
}

\begin{abstract}
The advection-dispersion model (ADM) is a good tool for simulating transport of dye or solutes in a solution conduit. Because the general problem of transport can be decomposed into two problems, a boundary-value problem and an initial-value problem, the complete solution is a superposition of the solutions for these two problems. In this paper, the solution for the general problem is explained. A direct application of the solution for the boundary-value problem is dye-tracing experiments. The purpose is inclusion of the input history of a solute dye into the ADM. The measured breakthrough curve of a dye-tracing experiment is used to invert for the release history of the dye at the input point through the ADM. It is mathematically shown that the breakthrough curve can not be directly used to invert for the boundary condition at a tracer release point. Therefore, a conductance-fitting method is employed to obtain the input history. The inverted history for a simple example is then shown to be a step function with amplitude of $420 \mu \mathrm{g} / \mathrm{L}$ and a duration of 10 minutes. Simulations illustrate that the breakthrough curves at downstream springs provide a means for understanding the migration of dye. A discussion of the implication of the solution for an initial-value problem (e.g., simulating transport of preexisting solutes such as dissolved calcium carbonate in solution conduits) is also included.
\end{abstract}

\section{INTRODUCTION}

Solute-transport modeling, as part of a quantitative tracer-test conducted in karstic aquifers, is well established to be critical to developing an understanding of the nature of solute migration in solution conduits (e.g., Field and Pinsky 2000; Birk et al. 2005). The essential solutetransport parameters of velocity, dispersion, and retardation, to name just a few, are generally determined from groundwater tracing and solute-transport modeling processes. Unfortunately, the general complexity of a tracerbreakthrough curve (BTC) often leads to the development or use of models of ever increasing intricacies in attempts to obtain improved model fits to measured data. Although the improved model fits are usually preferred and valuable, there remain some concerns as to the overall applicability of models with very large numbers of parameters, especially those whose parameters may not be transferrable to other examples.

The advection-dispersion model (ADM), also known as the equilibrium model, may be regarded as the simplest of the various mathematical models used to describe solute transport in solution conduits. Although the ADM is theoretically reasonable, its application to measured BTCs is often disappointing because of excessively skewed BTC tails in the measured data that cannot be matched using the ADM. The skewness is often attributed to strong exchanges between mobile- and immobile-flow regions (Toride et al. 1993), solute reactions with aquifer materials (Svensson and Dreybrodt 1992), or multiple flow paths.

Currently, the ADM and the two-region non-equilibrium model (2RNE) are two of the most popular models used for simulating solute transport in solution conduits (Toride et al., 1993; Field and Pinsky, 2000; Birk et al., 2005; Göppert and Goldscheider, 2008; Goldscheider, 2008). The $2 \mathrm{RNE}$ is advantageous in that excessive BTC skewness can be well simulated, but has the disadvantage of requiring additional parameters that lead to mathematical complications and possible errors in parameterization if local minima (as opposed to a global minimum) create a condition of non-uniqueness during inverse analysis (see, for example, Moré and Wright, 1993). The likelihood of a local minimum being encountered increases as the number of model parameters increases, especially if the initial value for one or more of the varying parameters is far from the real value.

The currently favored ADM is actually the solution for the boundary-value problem in which a zero initial condition is assumed such that the solute source is a selected boundary condition. In other words, it is the boundary condition that drives the BTCs, and that is most applicable to tracing experiments. In this paper, we first describe a complete solution that considers the contribution of the initial condition (similar to that of Toride et al., 1995, pp. 4-6). Second, we use a conductance-fitting method to obtain the input history of a dye tracing experiment.

\footnotetext{
* Corresponding Author

${ }^{1}$ U.S. Environmental Protection Agency, National Center for Environmental Assessment (8623P), 1200 Pennsylvania, Ave., N.W., Washington, D.C. 20460, USA, field.malcolm@epa.gov

Disclaimer: The views expressed in this paper are solely those of the authors and do not necessarily reflect the views or policies of the U.S. Environmental Protection Agency.

${ }^{2}$ Department of Geophysics, Yunnan University, 2 North Green Lake Rd., Kunming, Yunnan 650091, P. R. China, guangquanli74@gmail.com
} 
Finally, we discuss the difference of the ADM and the 2RNE in modeling transport in solution conduits.

\section{Advection-Dispersion Modeling in SOLUTION CONDUITS}

\section{Problem Formulation}

The essential features of solute transport in a solution conduit may be described using a one-dimensional advection-dispersion equation (Taylor, 1954), which arises from mass conservation of the solute, but water exchange between the solution conduit and the surrounding rock matrix is neglected. The governing equation of the general $\mathrm{ADM}$ is given as

$$
\frac{\partial C}{\partial t}+W \frac{\partial C}{\partial z}=D \frac{\partial^{2} C}{\partial z^{2}} .
$$

Solute concentration is denoted as $C$. The dispersion coefficient $D$ can be parameterized with the conduit radius, $a$, and solute velocity $W$ as follows (Li et al., 2008),

$$
D=\beta a W,
$$

where the dimensionless dispersion coefficient $\beta$ is used to quantify the strength of dispersion. We note that $\beta a$ is often referred to as dispersivity.

Because the length of the conduit is invariably finite, there is an initial condition within the solution conduit $(0 \leq z \leq L$, where $L$ is the downstream position of the spring),

$$
C(z, 0)=C_{\mathrm{I}}(z),
$$

and a boundary condition at the sinkhole $(z=0)$

$$
C(0, t)=C_{\mathrm{B}}(t) .
$$

The general problem consists of Equation (1) subject to conditions shown in Equations (3) and (4). Because the mathematical problem is linear with respect to concentration, we can decompose this general problem of the ADM into two problems; a boundary-value problem (BVP) and an initial-value problem (IVP) (Fig. 1). The solution is the superposition of the two solutions for these two problems.

\section{Solution for the Boundary-Value Problem}

The boundary-value problem consists of Equations (1) and (4), with a zero concentration initial condition, $C_{\mathrm{I}}(z)=0$. The Green's function for this problem can be obtained by the Laplace transform, and the solution is

$$
\begin{aligned}
C_{\mathrm{BVP}}(z, t)= & \int_{0}^{t} C_{\mathrm{B}}(\tau) \frac{z}{\sqrt{4 \pi D(t-\tau)^{3}}} \\
& \exp \left\{-\frac{[z-W(t-\tau)]^{2}}{4 D(t-\tau)}\right\} d \tau,
\end{aligned}
$$

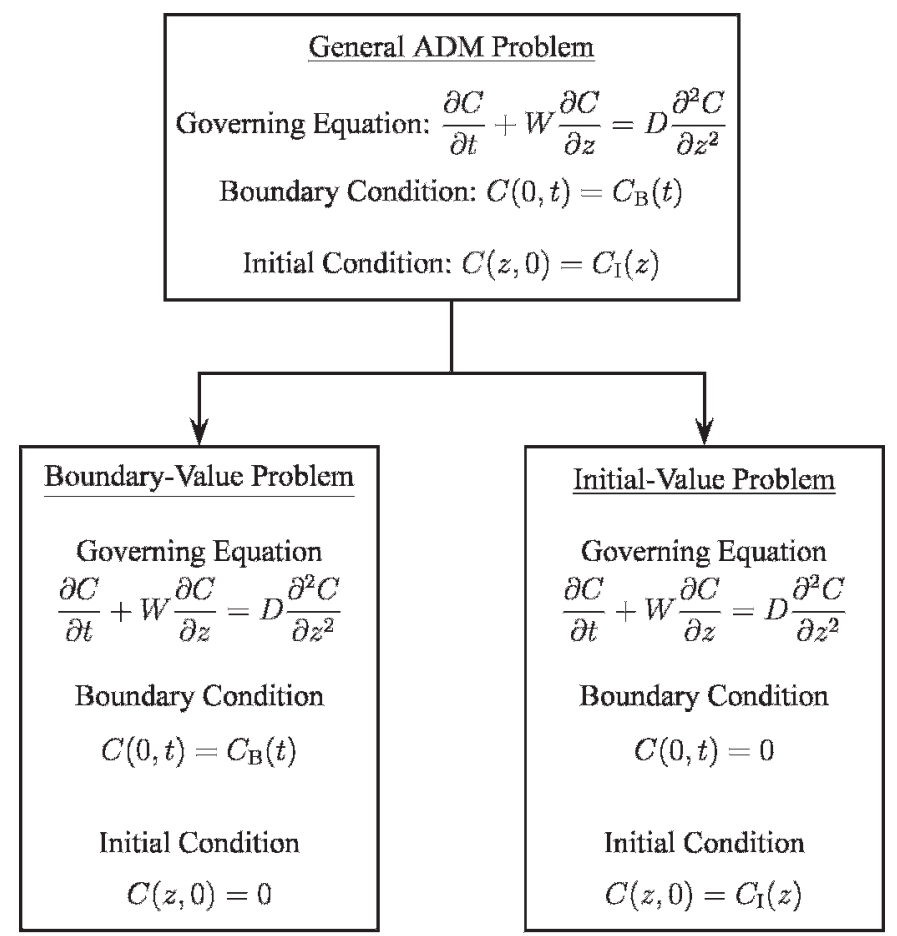

Figure 1. The schematic decomposition of the general problem of the ADM. The complete solution is a superposition of the solutions for each subproblem.

which is equivalent to the solution listed in Table 2 of Kreft and Zuber (1978) and Table 2.2 of Toride et al. (1995). Here, $\tau$ represents the past time, i.e., the time earlier than $t$.

\section{Solution for the Initial-Value Problem}

The solution for the initial-value problem is a convolution of the initial condition with the associated Green's function:

$$
\begin{aligned}
C_{\mathrm{IVP}}(z, t) & =\int_{0}^{L} C_{\mathrm{I}}(\zeta)\left\{\frac{1}{\sqrt{4 \pi D t}} \exp \left[\frac{-(z-W t-\zeta)^{2}}{4 D t}\right]\right. \\
& -\int_{0}^{t} \frac{1}{\sqrt{4 \pi D \tau}} \exp \left[\frac{-(W \tau+\zeta)^{2}}{4 D \tau}\right] \frac{z}{\sqrt{4 \pi D(t-\tau)^{3}}} \\
& \left.\times \exp \left(\frac{-[z-W(t-\tau)]^{2}}{4 D(t-\tau)}\right) d \tau\right\} d \zeta .
\end{aligned}
$$

Note that the term inside the big bracket is the Green's function for the initial-value problem. Here, $\zeta$ is a variable denoting the spatial coordinate of the solute-concentration distribution at $t=0$.

The Green's function for the initial-value problem in infinite space can be obtained from the Fourier transform, which is the first term inside the big bracket. However, this term causes a positive value at the boundary $z=0$. The initial-value problem requires a zero value at this location. 
Table 1. The parameters of the simulation example.

\begin{tabular}{lcl}
\hline Parameter & Value & Units \\
\hline Conduit radius, $a$ & 2.44 & $\mathrm{~m}$ \\
Conduit length, $L$ & 8.0 & $\mathrm{~km}^{-1}$ \\
Flow velocity, $W$ & 0.095 & $\mathrm{~m} \mathrm{~s}^{-1}$ \\
$\begin{array}{l}\text { Dimensionless dispersion } \\
\quad \text { coeff., } \beta\end{array}$ & 7.0 & $\ldots$ \\
$\quad$ Total mass of dye, $M$ & 476 & $\mathrm{gram}$ \\
$\quad \begin{array}{l}\text { Dye concentration at } \\
\quad \text { sinkhole, } C_{0}\end{array}$ & 420 & $\mu \mathrm{g} \mathrm{L}$ \\
$\quad \begin{array}{l}\text { Input duration at sinkhole, } \\
\quad T_{S T}\end{array}$ & 10 & $\min$ \\
$\quad$ Time sampling interval & 1 & $\mathrm{~min}$ \\
\hline
\end{tabular}

For this reason, a negative value must be prescribed at this boundary and the contribution of this negative boundary value must be included, which is the second term inside the big bracket. This Green's function (i.e., the term inside the big bracket) is equivalent to that listed in Table 2.2 of Toride et al. (1995).

\section{The Complete Solution}

The complete solution of Equation (1), subject to the initial and boundary conditions imposed by Equations (3) and (4), respectively is a superposition of solutions (5) and (6) that is given as

$$
C(z, t)=C_{\mathrm{BVP}}(z, t)+C_{\mathrm{IVP}}(z, t) .
$$

\section{ApPlicAtion}

This section applies Equation (5) for the boundaryvalue problem to a tracer experiment conducted at the Lost River Cave System located in Bowling Green, Kentucky. Formed in the Ste. Genevieve and St. Louis Limestones of Middle to Late Mississippian age and developed on top of the Lost River Chert, the Lost River Cave System begins with the Lost River flowing directly into the cave entrance. Lost River then traverses the cave to eventually reemerge $8 \mathrm{~km}$ downstream.

Flow along the length of the cave is relatively uniform along much of its length, but older, higher passages can be inundated by flood-flow conditions (Crawford, 1986, p. 7). Blocks of the Lost River Chert routinely detach from cave walls and line the cave floor as the limestone dissolves away. The detached chert blocks create minor detention backwaters containing immobile-flow regions. Undercut benches and recirculation with scalloped walls also serve as minor immobile flow regions.

The tracer input is modeled using a typical step-like function with time. By fitting the theoretical BTC against the measured BTC, the parameters of the solution conduit and solute transport, as well as the input history of tracer,

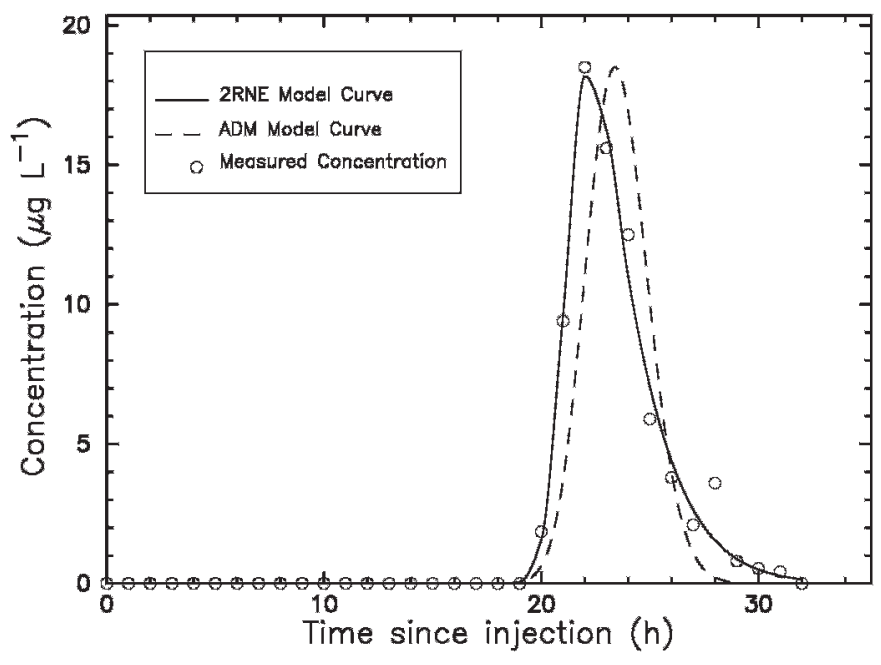

Figure 2. The theoretical breakthrough curves versus the measured curve. The initial condition is zero, and for the ADM, the boundary condition at the sinkhole is assumed to be a step-like function with time.

are inverted (Table 1). In our inversion process, the total mass of dye provides a constraint as follows:

$$
T_{S T}=\frac{M}{Q C_{0}},
$$

where $M$ is the total mass of dye (476 gram), $T_{S T}$ is the duration of dye input at the sinkhole, $C_{0}$ is the amplitude of dye concentration at the tracer release point, and $Q=\pi a^{2} W$ is the spring discharge $\left(1.78 \mathrm{~m}^{3} / \mathrm{s}\right)$. For the ADM, the boundary condition at the input point is assumed to be a step function with time (constrained by Equation (8)).

The 2RNE is also used to simulate the measured BTC. Both models (ADM and 2RNE) achieve a good fit against the measured BTC, as depicted in Fig. 2. The peak of the BTC modeled from ADM has an error of 2 hours (Fig. 2) because the ADM cannot adequately simulate the skewness of the measured BTC. The 2RNE visually appears to a better fit than the ADM in simulating the falling skewed limb.

Often, the dye release is assumed to be instantaneous (a Dirac- $\delta$ source). This assumption is reasonable as a firstorder approximation. However, this assumption mathematically implies an infinite solute-concentration at the injection moment. To overcome this physical problem, we include the input history into the ADM, and obtain a reasonable history of about 10 minutes. This result should be regarded as the second-order of approximation. Actually, because this duration is much shorter than the width of the measured BTC and the amplitude of solute concentration at the tracer release point $C_{0}\left(420 \mu \mathrm{g} \mathrm{L}^{-1}\right)$ is much larger than the maximum amplitude of the measured BTC at the spring $\left(18.5 \mu \mathrm{g} \mathrm{L}^{-1}\right)$, the continuous input could be regarded as a Dirac- $\delta$ source. For this reason a Dirac- $\delta$ source assumption often works well. Nevertheless, 
the continuous input is physically more reasonable, because concentration can never be infinitely large.

\section{Discussion}

For the inversion procedure, we use conductance-fitting, which is an indirect inversion method. Direct inversion may be thought of as using a negative velocity and a negative dispersion coefficient to infer the input history directly from the measured BTC. However, the Green's function for a Dirac- $\delta$ input at the spring does not exist. In a physical sense, a point source at the spring would be restored to an input history that never exists. Mathematically, this can be shown by rewriting Equation (5) to

$$
C_{\mathrm{BVP}}(z, t)=C_{\mathrm{B}}(t) * G_{\mathrm{B}}(z, t),
$$

where $*$ represents convolution. Performing the Laplace transform with respect to time on Equation (9) yields

$$
C_{\mathrm{B}}(p)=\frac{C_{\mathrm{BVP}}(z, p)}{G_{\mathrm{B}}(z, p)},
$$

and applying an inverse Laplacian transform and convolution on Equation (10) yields

$$
C_{\mathrm{B}}(t)=C_{\mathrm{BVP}}(z, t) * L T^{-1}\left[\frac{1}{G_{\mathrm{B}}(z, p)}\right],
$$

where $L T^{-1}$ denotes inverse Laplacian transform. The inverse Laplacian transform of the reciprocal of $G_{\mathrm{B}}(z, p)$ doesn't exist. Therefore, we cannot invert for the input history $C_{\mathrm{B}}(t)$ directly from the spring $\mathrm{BTC} C_{\mathrm{BVP}}(z, t)$, and the conductance-fitting must be used.

The ADM is often applied to tracer experiments in karstic aquifers for which the initial condition is zero, but the boundary condition (history of the tracer solute) at the input point is not zero. Besides tracing experiments, the ADM is also applicable to the simulation of transport in which the boundary condition is zero, but the initial condition is not zero. Such a situation may be encountered when water entering a sinkhole or similar input point is tracer free, but water rich in dissolved solutes (e.g., calcium carbonate) preexists inside the conduit. Because the initial condition is often unknown and not measurable, the distribution of solutes in the solution conduit must be assumed a priori.

Toride et al. (1993) proposed a 2RNE model, to describe nonequilibrium solute transport with first-order decay and zero-order production. The ADM model could be seen as a simplification of their model. The Toride et al. model dealt with the case in which there is a strong solute interaction between mobile- and immobile-flow regions or aquifer kinetic reactions, while our model focuses on the case in which aquifer kinetic reactions and solute interac- tion between mobile- and immobile-flow regions are negligible. Our solution to the ADM may be regarded as more robust, because it requires fewer parameters, which is similar to the findings of Göppert and Goldscheider (2008). In this sense, our model may be more suitable to the relatively rapid transport conditions that typically occur in solution conduits, because when solutes are rapidly flushed and entrained through the solution conduit, only a small portion of the solute will likely be sequestered in an immobile-flow region ( $\mathrm{Li}$ et al., 2008) and probably only for a very short time. Therefore, the solute interaction between the mobile- and immobile-flow regions should be very limited, and our model should behave very well as a first-order approximation. Nevertheless, the 2RNE model provides a more accurate tool, because it considers temporary solute detention in immobile-flow regions. For this reason, the $2 \mathrm{RNE}$ is better for simulating BTCs that exhibit significant skewness and long tailing phenomena (see Birk et al., 2005), while the ADM is suitable for simulating the primary large signals that arrive earlier.

\section{Summary AND Conclusion}

Transport in a solution conduit is often described by the advection-dispersion model that is subjected to various initial and boundary conditions. The general problem is a superposition of two solutions: one is for the problem consisting of a boundary condition and a zero initial condition (Equation (5)), and the other is for the problem consisting of an initial condition and a zero boundary condition (Equation (6)).

We used Equation (5) and the breakthrough curve measured from a dye tracing experiment to invert for the history of dye injection at the input point. The inverted parameters are reasonable, which illustrates that the BTCs can be used to obtain the history of solute injection at input points. From Figure 2, the breakthrough curve of the boundary-value problem still exhibits some skewness. In this sense, the 2RNE is a better model that better replicates the typically strong skewness and tailing observed in measured BTCs. It is our contention that the ADM will have important and profound applications in modeling transport of solutes that preexist in solution conduits (e.g., dissolved carbonates).

\section{ACKNOWLEDGEMENTS}

This research was financially supported in part by the University Fund of Yunnan University under contract KL090020. The authors are deeply grateful to the Associate Editor, Prof. Gregory S. Springer for his insightful comments and constructive suggestions. 


\section{NotATion}

\begin{tabular}{|c|c|}
\hline & conduit radius $(\mathrm{m})$ \\
\hline$C, C(z, t)$ & solute concentration in conduit $\left(\mathrm{g} \mathrm{L}^{-1}\right)$ \\
\hline$C_{0}$ & concentration of dye at sinkhole $\left(\mathrm{g} \mathrm{L}^{-1}\right)$ \\
\hline$C_{\mathrm{B}}(t)$ & boundary condition at sinkhole $\left(\mathrm{g} \mathrm{L}^{-1}\right)$ \\
\hline$C_{\mathrm{BVP}}(z, t)$ & $\begin{array}{l}\text { solute concentration for the boundary-value } \\
\text { problem }\left(\mathrm{g} \mathrm{L}^{-1}\right)\end{array}$ \\
\hline$C_{\mathrm{I}}(t)$ & initial condition within conduit $\left(\mathrm{g} \mathrm{L}^{-1}\right)$ \\
\hline$C_{\mathrm{IVP}}(z, t)$ & $\begin{array}{l}\text { solute concentration for the initial-value prob- } \\
\text { lem }\left(\mathrm{g} \mathrm{L}^{-1}\right)\end{array}$ \\
\hline$D$ & dispersion coefficient $\left(\mathrm{m}^{2} \mathrm{~s}^{-1}\right)$ \\
\hline$G_{\mathrm{B}}(z, t)$ & $\begin{array}{l}\text { Green's function for the boundary-value prob- } \\
\text { lem with source fixed at } t=0\left(\mathrm{~s}^{-1}\right)\end{array}$ \\
\hline$L$ & conduit length (m) \\
\hline$M$ & total mass of dye (gram) \\
\hline$p$ & Laplacian transform variable $\left(\mathrm{s}^{-1}\right)$ \\
\hline$Q$ & water discharge $\left(\mathrm{m}^{3} \mathrm{~s}^{-1}\right)$ \\
\hline$t$ & time $(\mathrm{s})$ \\
\hline $\begin{array}{l}T_{S T} \\
W\end{array}$ & $\begin{array}{l}\text { input duration of dye at sinkhole, } \\
\text { flow velocity in conduit }\left(\mathrm{m} \mathrm{s}^{-1}\right)\end{array}$ \\
\hline$z$ & $\begin{array}{l}\text { conduit downstream location starting from } \\
\text { sinkhole }(\mathrm{m})\end{array}$ \\
\hline$\beta$ & dimensionless dispersion coefficient \\
\hline
\end{tabular}

Birk, S., Geyer, T., Liedl, R., and Sauter, M., 2005, Process-based interpretation of tracer tests in carbonate aquifers: Ground Water, v. 43 , no. 3 , p. $381-388$, doi: $10.1111 /$ j.1745-6584.2005.0033.x.
Crawford, N.C., 1986, Karst hydrologic problems associated with urban development: ground water contamination, hazardous fumes, sinkhole flooding, and sinkhole collapse in the Bowling Green area, Kentucky, Guide Book to Field Trip B: Environmental Problems in Karst Terranes and Their Solutions Conference (Bowling Green): National Water Well Association, Dublin, Ohio, 85 p.

Field, M.S., and Pinsky, P.F., 2000, A two-region nonequilibrium model for solute transport in solution conduits in karstic aquifers: Journal of Contaminant Hydrology, v. 44, no. 3-4, p. 329-351.

Goldscheider, N., 2008, A new quantitative interpretation of the long-tail and plateau-like breakthrough curves from tracer tests in the artesian karst aquifer of Stuttgart, Germany: Hydrogeology Journal, v. 16, p. 1311-1317, doi: 10.1007/s10040-008-0307-0.

Göppert, N., and Goldscheider, N., 2008, Solute and Colloid Transport in Karst Conduits under Low- and High-Flow Conditions: Ground Water, v. 46, no. 1, p. 61-68, doi: 10.1111/j.1745-6584.2007.00373.x.

Kreft, A., and Zuber, A., 1978, On the physical meaning of dispersion equation and its solution for different initial and boundary conditions: Chemical Engineering Science, v. 33, no. 11, p. 1471-1480.

Li, G., Loper, D.E., and Kung, R., 2008, Contaminant sequestration in karstic aquifers: Experiments and quantification: Water Resources Research, v. 44, W02429, doi: 10.1029/2006WR005797.

Moré, J.J., and Wright, S.J., 1993, Optimization software guide: Philadelphia, Society for Industrial and Applied Mathematics, $154 \mathrm{p}$.

Svensson, U., and Dreybrodt, W., 1992, Dissolution kinetics of natural calcite minerals in $\mathrm{CO}_{2}$-water systems approaching calcite equilibrium: Chemical Geology, v. 100, no. 1-2, p. 129-145.

Taylor, G.I., 1954, The dispersion of matter in turbulent flow through a pipe: Proceedings of the Royal Society London, Ser. A, v. 223, p. 446-468, doi: 10.1098/rspa.1954.0130.

Toride, N., Leij, F.J., and van Genuchten, M.T., 1993, A comprehensive set of analytical solutions for nonequilibrium solute transport with first-order decay and zero-order production: Water Resources Research, v. 29, no. 7, p. 2167-2182, doi: 10.1029/93WR00496.

Toride, N., Leij, F.J., and van Genuchten, M.T., 1995, The CXTFIT code for estimating transport parameters from the laboratory or field tracer experiments; version 2.0: US Salinity Laboratory Research Report $137,121 \mathrm{p}$. 